\title{
Uniform Order Continuous Block Hybrid Method for the Solution of First Order Ordinary Differential Equations
}

\author{
${ }^{1}$ A.A. James,${ }^{2}$ A.O. Adesanya, ${ }^{3}$ J. Sunday \\ ${ }^{1}$ Mathematics Division, American University of Nigeria, Yola, Adamawa State, Nigeria \\ ${ }^{2}$ Department of Mathematics, ModibboAdama University of Technology, Yola, Nigeria. \\ ${ }^{3}$ Department of Mathematical Sciences, Adamawa State University, Mubi, Nigeria.
}

\begin{abstract}
We know that for any numerical method to be efficient and computational reliable, it must be convergent, consistent, and stable. This paper adopted the method of interpolation of the approximate solution and collocation ofits differential system at grid and off grid points to yield a continuous linear multistep method with a constant step size. The continuous linear multistep method is solved for the independent solution to yield a continuous block method which is evaluated at selected grid and off grid points to yield a discrete block method. The basic property of this method is verified to be convergent consistent and satisfies the conditions for stability. The method was tested on numerical examples and found to compete favorably withthe existing methods in term of accuracy and error variation.
\end{abstract}

Keywords: interpolation, IVP, ODEs, colocation, approximate solution, independent solution, block method, convergent.

\section{INTRODUCTION}

It has been established that a given linear or non-linear equations does not have a complete solution that can be expressed in terms of a finite number of elementary functions (Ross. 1964; Humi and Miller, 1988). It has also been established that such problems could be solved by seeking an approximate solution by adopting interpolation and collocation method.

In this paper, we consider a numerical method for solving first order initial value problems of the form

$$
y^{\prime}=f(x, y), \quad y\left(x_{0}\right)=y_{0}
$$

Many scholars have worked on the development of a continuous linear multistep in finding solution to (1). These authors proposed method with different basis functions, among them are Serisena, Onumanyi and Chollom (2001), Awoyemi, Ademiluyi and Amuseghan (2008), Ikhile (2008), Adeniyi, Deyefa and Alabi (2006), Fatokun, Onumanyi and Serisena (2005), Badmus and Mishelia (2011), Olorunsola and Enoch (2011), Umaru (2011), Yahaya and Kumlemg (2007), Ibijola, Skwane and Kumleng (2011), James et, al (2012), Adesanya, Odekunle, and James (2012) to mention few. These authors proposed method ranging from predictor corrector method to discrete block method.

In this paper, we propose a continuous block method which when evaluated at selected grid points gives a discrete block which the authors mentioned above had proposed. The continuous block possesses the same properties as the continuous linear multistep method. This paper is partitioned into sections as follows: Section two is methodology involved in deriving the continuous multistep method and the continuous block method. Section three considers the analysis of the block method viz; the order, zero stability and the region of absolute stability. Section four considers the numerical examples where we test our method on first order ordinary differential equation and compare our result with existing methods.

\section{MeTHODOLOGY}

Consider a monomial power approximate solution in the form

$$
y(x)=\sum_{j=0}^{s+r-1} a_{j} x^{j}
$$

where $\mathrm{r}$ and $\mathrm{s}$ are interpolation and collocation points respectively. The first derivative of (2) gives

$$
y^{\prime}(x)=\sum_{j=0}^{s+r-1} j a_{j} x^{j-1}
$$

Substituting (3) into (1) gives

$$
f(x, y)=\sum_{j=0}^{s+r-1} j a_{j} x^{j-1}
$$


collocating (4) at $x_{n+s}, s=0\left(\frac{1}{12}\right) 1$ and interpolating (2) at $x_{n}$ gives and a system of non-linear equation in the form

where

$$
A X=U
$$

$A=\left[a_{0}, a_{1}, a_{2}, a_{3}, a_{4}, a_{5}, a_{6}, a_{7}, a_{8} a_{9}, a_{10}, a_{11} a_{12}, a_{13}\right]^{T}$

$X$

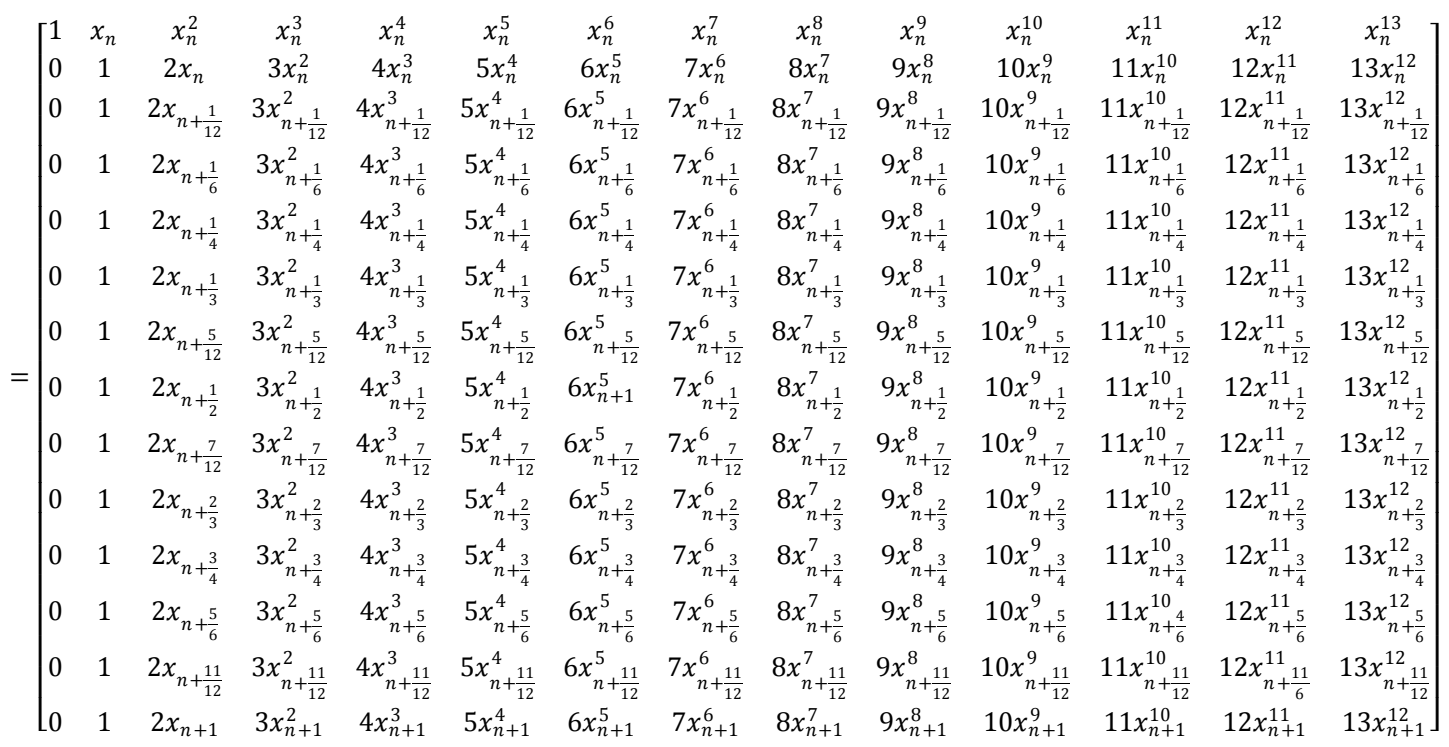

Solving (5) for the $a_{j} s$ and substituting back into (2) gives a continuous multistep method in the form

$$
y(x)=\alpha_{0} y_{n}+h \sum_{j=0}^{13} \beta_{j}(x) f_{n+j}
$$

Where $\alpha_{0}=1$ and the coefficients of $f_{n+j}$ gives

$\beta_{0}=(\mathrm{b}$

$$
\begin{aligned}
&\left.\frac{1}{63063000}\right)\left(90296156160 \mathrm{t}^{13}-635835432960 \mathrm{t}^{12}+2013478871040 \mathrm{t}^{11}-3788519454720 \mathrm{t}^{10}\right. \\
&+ 4714062312960 \mathrm{t}^{9}-4084497537120 \mathrm{t}^{8}+2526651372960 \mathrm{t}^{7}-1125143019000 \mathrm{t}^{6} \\
&+\left.358834932456 \mathrm{t}^{5}-80422096755 \mathrm{t}^{4}+12206383280 \mathrm{t}^{3}-1174186650 \mathrm{t}^{2}+63063000 \mathrm{t}\right)
\end{aligned}
$$

$$
\begin{aligned}
\beta_{\frac{1}{12}}=\left(-\frac{1}{875875}\right) & \left(15049359360 t^{13}-104613949440 t^{12}+326069452800 t^{11}-601530209280 t^{1^{0}}\right. \\
+ & 729979810560 t^{9}-612313982280 t^{8}+362792944800 t^{7}-152252300200 t^{6} \\
+ & \left.44580592056 t^{5}-8759871120 t^{4}+1061078200 t^{3}-63063000 t^{2}\right) \\
\beta_{\frac{1}{6}}=\left(\frac{1}{875875}\right)(82 & 771476480 t^{13}-567904296960 t^{12}+1742433638400 t^{11}-3153363333120 t^{1^{0}} \\
+ & 3737267614080 t^{9}-3043385064720 t^{8}+1736404698600 t^{7}-693746853800 t^{6} \\
+ & \left.190182650658 t^{5}-34098869805 t^{4}+3611658050 t^{3}-173423250 t^{2}\right)
\end{aligned}
$$

$$
\begin{aligned}
\beta_{\frac{1}{4}=}\left(-\frac{1}{1576575}\right) & \left(496628858880 t^{13}-3362591232000 t^{12}+10157063454720 t^{11}-18043664550912 t^{1^{0}}\right. \\
& +20915213679360 t^{9}-16582332606840 t^{8}+9158773235040 t^{7}-3516977744280 t^{6} \\
& \left.+918498805224 t^{5}-155290655520 t^{4}+15371556200 t^{3}-693693000 t^{2}\right)
\end{aligned}
$$

$\beta_{\frac{1}{3}}=\left(\frac{1}{1401400}\right)\left(993257717760 t^{13}-6635513364480 t^{12}+19735353630720 t^{11}-34437417670656 t^{1^{0}}\right.$

$+39100086305280 t^{9}-30266940543840 t^{8}+16262902038240 t^{7}-6052111305240 t^{6}$

$\left.+1526339734920 t^{5}-248668174755 t^{4}+23751027300 t^{3}-1040539500 t^{2}\right)$

$\beta_{\frac{5}{21}}=\left(\frac{1}{875875}\right)\left(993257717760 t^{13}-6545844264960 t^{12}+19172883824640 t^{11}-32886142248960 t^{1^{0}}\right.$

$+36629619586560 t^{9}-27759338727120 t^{8}+14574432375360 t^{7}-5291779693200 t^{6}$

$\left.+1301294410416 t^{5}-206885558880 t^{4}+19333794480 t^{3}-832431600 t^{2}\right)$

$\beta_{\frac{1}{2}}=\left(\frac{1}{3753755}\right)\left(496628858880 t^{13}-3228087582720 t^{12}+9313358745600 t^{11}-15714509690880 t^{1^{0}}\right.$

$+17197059559680 t^{9}-12791390451840 t^{8}+6587216578800 t^{7}-2345743600200 t^{6}$

$\left.+566145968388 t^{5}-88480301910 t^{4}+8148240100 t^{3}-346846500 t^{2}\right)$ 
$\beta_{\frac{7}{12}}=\left(-\frac{1}{875875}\right)\left(993257717760 t^{13}-6366506065920 t^{12}+18096854630400 t^{11}-30061565614080 t^{1^{0}}\right.$ $+32370337359360 t^{9}-23686439016240 t^{8}+12002205758400 t^{7}-4208405401200 t^{6}$ $\left.+1001300476176 t^{5}-154528133760 t^{4}+14081667600 t^{3}-594594000 t^{2}\right)$

$\beta_{\frac{2}{3}}=\left(\frac{1}{1401400}\right)\left(993257717760 t^{13}-6276836966400 t^{12}+17583295242240 t^{11}-28779297490944 t^{1^{0}}\right.$ $+30536687301120 t^{9}-22026957432480 t^{8}+11010721344480 t^{7}-3812678829960 t^{6}$ $\left.+897041193048 t^{5}-137108736765 t^{4}+12395783400 t^{3}-520269750 t^{2}\right)$

$\beta_{\frac{3}{4}}=\left(-\frac{1}{1576575}\right)\left(496628858880 t^{13}-3093583933440 t^{12}+8543019663360 t^{11}-13788865778688 t^{1^{0}}\right.$ $+14436621239040 t^{9}-10283837283720 t^{8}+5081864879520 t^{7}-1741654834920 t^{6}$ $\left.+406102777080 t^{5}-61600178640 t^{4}+5534929400 t^{3}-231231000 t^{2}\right)$

$\beta_{\frac{5}{6}}=\left(\frac{1}{875875}\right)\left(82771476480 t^{13}-508124897280 t^{12}+1383757240320 t^{11}-2204365363200 t^{1^{0}}\right.$ $+2280144746880 t^{9}-1606487042160 t^{8}+786109432680 t^{7}-267108041200 t^{6}$ $\left.+61823980218 t^{5}-9320275965 t^{4}+833322490 t^{3}-34684650 t^{2}\right)$

$\beta_{\frac{11}{12}}=\left(-\frac{1}{875875}\right)\left(15049359360 t^{13}-91027722240 t^{12}+244552089600 t^{11}-384829885440 t^{1^{0}}\right.$ $+393720687360 t^{9}-274725130680 t^{8}+133301282400 t^{7}-44965520600 t^{6}$ $\left.+10343749416 t^{5}-1551469920 t^{4}+138156200 t^{3}-5733000 t^{2}\right)$

$\beta_{1}=\left(\frac{1}{63063000}\right)$ $\left(90296156160 t^{13}-538014597120 t^{12}+1426553856000 t^{11}-2219310213120 t^{1^{0}}\right.$ $+2248162076160 t^{9}-1555315201440 t^{8}+749148285600 t^{7}-251136685800 t^{6}$ $\left.+57470909496 t^{5}-8583459885 t^{4}+761770100 t^{3}-31531500 t^{2}\right)$

Where $t=\frac{x-x_{n}}{h}$. Solving (6) for the independent solution gives a continuous block method in the form

$$
y_{n+k}=\sum_{j=0}^{\mu-1} \frac{(j h)^{m}}{m !} y_{n}^{(m)}+h^{\mu} \sum_{j=0}^{s} \sigma_{j}(x) f_{n+j}
$$

Where $\mu$ is the order of the differential equation, $s$ is the collocation points. Hence the coefficient of $f_{n+j}$ in (7) $\sigma_{0}=(\mathrm{b}$

$$
\begin{aligned}
&\left.\frac{1}{63063000}\right)\left(90296156160 \mathrm{t}^{13}-635835432960 \mathrm{t}^{12}+2013478871040 \mathrm{t}^{11}-3788519454720 \mathrm{t}^{0}\right. \\
&+ 4714062312960 \mathrm{t}^{9}-4084497537120 \mathrm{t}^{8}+2526651372960 \mathrm{t}^{7}-1125143019000 \mathrm{t}^{6} \\
&\left.+358834932456 \mathrm{t}^{5}-80422096755 \mathrm{t}^{4}+12206383280 \mathrm{t}^{3}-1174186650 \mathrm{t}^{2}+63063000 \mathrm{t}\right)
\end{aligned}
$$

$\sigma_{\frac{1}{12}}=\left(-\frac{1}{875875}\right)\left(15049359360 t^{13}-104613949440 t^{12}+326069452800 t^{11}-601530209280 t^{1^{0}}\right.$ $+729979810560 t^{9}-612313982280 t^{8}+362792944800 t^{7}-152252300200 t^{6}$ $\left.+44580592056 t^{5}-8759871120 t^{4}+1061078200 t^{3}-63063000 t^{2}\right)$

$\sigma_{\frac{1}{6}}=\left(\frac{1}{875875}\right)\left(82771476480 t^{13}-567904296960 t^{12}+1742433638400 t^{11}-3153363333120 t^{t^{0}}\right.$ $+3737267614080 t^{9}-3043385064720 t^{8}+1736404698600 t^{7}-693746853800 t^{6}$ $\left.+190182650658 t^{5}-34098869805 t^{4}+3611658050 t^{3}-173423250 t^{2}\right)$

$\sigma_{\frac{1}{4}}=\left(-\frac{1}{1576575}\right)\left(496628858880 t^{13}-3362591232000 t^{12}+10157063454720 t^{11}-18043664550912 t^{1^{0}}\right.$ $+20915213679360 t^{9}-16582332606840 t^{8}+9158773235040 t^{7}-3516977744280 t^{6}$ + $\left.918498805224 t^{5}-155290655520 t^{4}+15371556200 t^{3}-693693000 t^{2}\right)$

$\sigma_{\frac{1}{3}}=\left(\frac{1}{1401400}\right)\left(993257717760 t^{13}-6635513364480 t^{12}+19735353630720 t^{11}-34437417670656 t^{1^{0}}\right.$ $+39100086305280 t^{9}-30266940543840 t^{8}+16262902038240 t^{7}-6052111305240 t^{6}$ $\left.+1526339734920 t^{5}-248668174755 t^{4}+23751027300 t^{3}-1040539500 t^{2}\right)$ $\sigma_{\frac{5}{21}}=\left(\frac{1}{875875}\right)\left(993257717760 t^{13}-6545844264960 t^{12}+19172883824640 t^{11}-32886142248960 t^{1^{0}}\right.$ $+36629619586560 t^{9}-27759338727120 t^{8}+14574432375360 t^{7}-5291779693200 t^{6}$ $\left.+1301294410416 t^{5}-206885558880 t^{4}+19333794480 t^{3}-832431600 t^{2}\right)$ $\sigma_{\frac{1}{2}}=\left(\frac{1}{3753755}\right)\left(496628858880 t^{13}-3228087582720 t^{12}+9313358745600 t^{11}-15714509690880 t^{1^{0}}\right.$ $+17197059559680 t^{9}-12791390451840 t^{8}+6587216578800 t^{7}-2345743600200 t^{6}$ $\left.+566145968388 t^{5}-88480301910 t^{4}+8148240100 t^{3}-346846500 t^{2}\right)$ 


$$
\begin{aligned}
& \sigma_{\frac{7}{12}}=\left(-\frac{1}{875875}\right)\left(993257717760 t^{13}-6366506065920 t^{12}+18096854630400 t^{11}-30061565614080 t^{1^{0}}\right. \\
& +32370337359360 t^{9}-23686439016240 t^{8}+12002205758400 t^{7}-4208405401200 t^{6} \\
& \left.+1001300476176 t^{5}-154528133760 t^{4}+14081667600 t^{3}-594594000 t^{2}\right) \\
& \sigma_{\frac{2}{3}}=\left(\frac{1}{1401400}\right)\left(993257717760 t^{13}-6276836966400 t^{12}+17583295242240 t^{11}-28779297490944 t^{1^{0}}\right. \\
& +30536687301120 t^{9}-22026957432480 t^{8}+11010721344480 t^{7}-3812678829960 t^{6} \\
& \left.+897041193048 t^{5}-137108736765 t^{4}+12395783400 t^{3}-520269750 t^{2}\right) \\
& \sigma_{\frac{3}{4}}=\left(-\frac{1}{1576575}\right)\left(496628858880 t^{13}-3093583933440 t^{12}+8543019663360 t^{11}-13788865778688 t^{1^{0}}\right. \\
& +14436621239040 t^{9}-10283837283720 t^{8}+5081864879520 t^{7}-1741654834920 t^{6} \\
& \left.+406102777080 t^{5}-61600178640 t^{4}+5534929400 t^{3}-231231000 t^{2}\right) \\
& \sigma_{\frac{5}{6}}=\left(\frac{1}{875875}\right)\left(82771476480 t^{13}-508124897280 t^{12}+1383757240320 t^{11}-2204365363200 t^{10}\right. \\
& +2280144746880 t^{9}-1606487042160 t^{8}+786109432680 t^{7}-267108041200 t^{6} \\
& \left.+61823980218 t^{5}-9320275965 t^{4}+833322490 t^{3}-34684650 t^{2}\right) \\
& \sigma_{\frac{11}{12}}=\left(-\frac{1}{875875}\right)\left(15049359360 t^{13}-91027722240 t^{12}+244552089600 t^{11}-384829885440 t^{t^{0}}\right. \\
& +393720687360 t^{9}-274725130680 t^{8}+133301282400 t^{7}-44965520600 t^{6} \\
& \left.+10343749416 t^{5}-1551469920 t^{4}+138156200 t^{3}-5733000 t^{2}\right) \\
& \sigma_{1}=\left(\frac{1}{63063000}\right)(90 \\
& \left(90296156160 t^{13}-538014597120 t^{12}+1426553856000 t^{11}-2219310213120 t^{1^{0}}\right. \\
& +2248162076160 t^{9}-1555315201440 t^{8}+749148285600 t^{7}-251136685800 t^{6} \\
& \left.+57470909496 t^{5}-8583459885 t^{4}+761770100 t^{3}-31531500 t^{2}\right)
\end{aligned}
$$

wheret $=\frac{x-x_{n}}{h}$. Evaluating (7) at $t=\frac{1}{12}\left(\frac{1}{12}\right) 1$ gives a discrete block formula of the form

$$
Y_{m}=e y_{n}+h d f\left(y_{n}\right)+h d f\left(Y_{m}\right)
$$

where e, d, are rx r matrix

$d=\left[\frac{703604254357}{313841848320000} \quad \frac{5389909963}{245188944000} \quad \frac{2846527447}{2846527447} \quad \frac{337524401}{337524401} \quad \frac{337524401}{251073478656} \quad \frac{22226233}{1009008000}\right.$ $\left.\begin{array}{llllll}14110554661 & 42194069 & 316182879 & 43189735 & 62984859487 & 1364651\end{array}\right]^{T}$

$\overline{640493568000} \frac{1915538625}{14350336000} \frac{1961511552}{2853107712000} \frac{1364063000}{630}$

Where

$$
\begin{gathered}
Y_{m}=\left[y_{n+\frac{1}{12}}, y_{n+\frac{1}{6}}, y_{n+\frac{1}{4},} y_{n+\frac{1}{3}}, y_{n+\frac{5}{12}} y_{n+\frac{1}{2}}, y_{n+\frac{7}{12}}, y_{n+\frac{2}{3}}, y_{n+\frac{3}{4}}, y_{n+\frac{5}{6}} y_{n+\frac{11}{12}}, y_{n+1}\right]^{T} \\
e=\left[\begin{array}{cccccccccccccc}
0 & 0 & 0 & 0 & 0 & 0 & 0 & 0 & 0 & 0 & 0 & 1 \\
0 & 0 & 0 & 0 & 0 & 0 & 0 & 0 & 0 & 0 & 0 & 1 \\
0 & 0 & 0 & 0 & 0 & 0 & 0 & 0 & 0 & 0 & 0 & 1 \\
0 & 0 & 0 & 0 & 0 & 0 & 0 & 0 & 0 & 0 & 0 & 1 \\
0 & 0 & 0 & 0 & 0 & 0 & 0 & 0 & 0 & 0 & 0 & 1 \\
0 & 0 & 0 & 0 & 0 & 0 & 0 & 0 & 0 & 0 & 0 & 1 \\
0 & 0 & 0 & 0 & 0 & 0 & 0 & 0 & 0 & 0 & 0 & 1 \\
0 & 0 & 0 & 0 & 0 & 0 & 0 & 0 & 0 & 0 & 0 & 1 \\
0 & 0 & 0 & 0 & 0 & 0 & 0 & 0 & 0 & 0 & 0 & 1 \\
0 & 0 & 0 & 0 & 0 & 0 & 0 & 0 & 0 & 0 & 0 & 1 \\
0 & 0 & 0 & 0 & 0 & 0 & 0 & 0 & 0 & 0 & 0 & 1 \\
0 & 0 & 0 & 0 & 0 & 0 & 0 & 0 & 0 & 0 & 0 & 1 \\
0 & 0 & 0 & 0 & 0 & 0 & 0 & 0 & 0 & 0 & 0 & 1
\end{array}\right]
\end{gathered}
$$


Uniform Order Continuous Block Hybrid Method for the Solution of First Order Ordinary

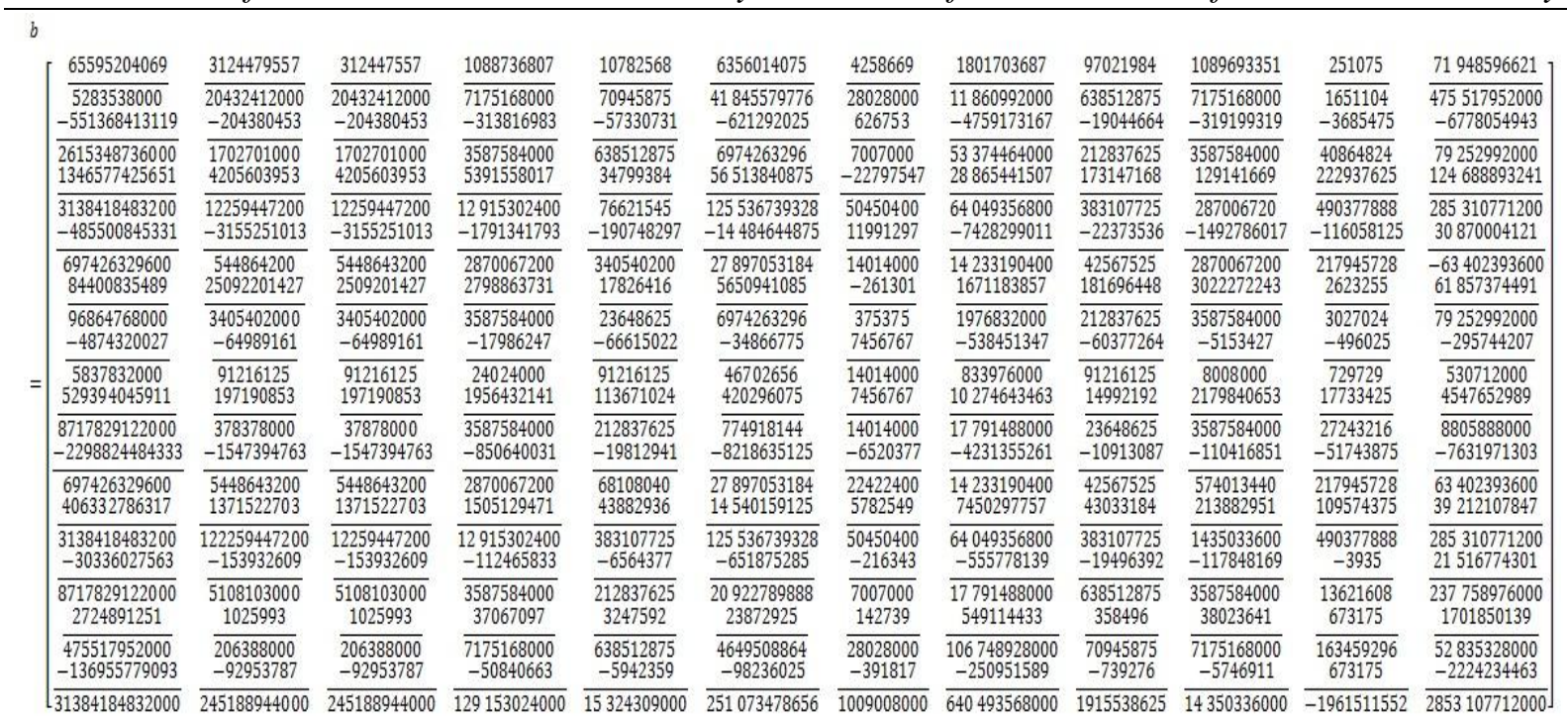

\section{Analysis Of The Basic Properties Of The NeW Block Method \\ ORDER OF THE METHOD}

Let the linear operator $L\{y(x): h\}$ associated with the block formula be defined as

$$
L\{y(x): h\}=A^{(0)} Y_{m}-e y_{n}-h^{\mu} d f\left(y_{n}\right)-h^{\mu} b F\left(Y_{m}\right)
$$

expanding in Taylor series expansion and comparing the coefficient of $\mathrm{h}$ gives

$$
L\{y(x) ; h\}=c_{0} y(x)+c_{1} h y^{\prime}(x)+c_{1} h y^{\prime \prime}(x) \ldots c_{p} h^{p} y^{p}(x)+c_{p+1} h^{p+1} y^{p+1}(x)+c_{p+2} h^{p+2} y^{p+2}
$$

Definition:

The linear operator $\mathrm{L}$ and the associated continuous linear multistep method (9) are said to be of order $\mathrm{p}$ if $c_{0}=c_{1}=c_{2} \ldots=c_{p}=0$ and $c_{p+1} \neq 0$ is called the error constant and implies that the local truncation error is given by $t_{n+k}=C_{p+1} \mathrm{~h}^{\mathrm{p}+1} \mathrm{y}^{\mathrm{p}+1}\left(\mathrm{x}_{n}\right)+0\left(\mathrm{~h}^{\mathrm{p}+2}\right)$.For our method, Expanding in Taylor series expansion gives and equating coefficients of the Taylor series expansion to zero yield a constant order 13 with the following error constants

$$
\begin{aligned}
c_{0}=c_{1}=\cdots c_{12} & =0, c_{14} \\
& =[-2.659(-11)-2.5088(-11)-2.4920(-11)-2.495(-11)-2.4941(-11) \\
& -2.4944(-11)-2.494(-11)-2.4984(-11)-2.4887(-11)-2.45485(-11) \\
& -2.3367(-11) \ldots]^{T}
\end{aligned}
$$

\section{Zero Stability}

Definition: The block (8)is said to be zero stable, if the roots $Z s, s=1,2 \ldots, N$ of the characteristic polynomial $\rho(z)$ defined by $\rho(z) \operatorname{det}\left(z A^{(0)}-E\right)$ satisfies $\left|z_{s}\right| \leq 1$ and every root satisfying $\left|z_{s}\right| \leq 1$ have multiplicity not exceeding the order of the differential equation. Moreover ash $\rightarrow 0, \rho(z)=z^{r-\mu}(z-1)^{\mu}$ where $\mu$ is the order of the differential equation, $r$ is the order of the matrix $A^{(0)}$ and $E$ (see Awoyemi et al.[6] for details).

For our method

$$
\left.\rho(z)=\left[\begin{array}{llllllllllll}
1 & 0 & 0 & 0 & 0 & 0 & 0 & 0 & 0 & 0 & 0 & 0 \\
0 & 1 & 0 & 0 & 0 & 0 & 0 & 0 & 0 & 0 & 0 & 0 \\
0 & 0 & 1 & 0 & 0 & 0 & 0 & 0 & 0 & 0 & 0 & 0 \\
0 & 0 & 0 & 1 & 0 & 0 & 0 & 0 & 0 & 0 & 0 & 0 \\
0 & 0 & 0 & 0 & 1 & 0 & 0 & 0 & 0 & 0 & 0 & 0 \\
0 & 0 & 0 & 0 & 0 & 1 & 0 & 0 & 0 & 0 & 0 & 0 \\
0 & 0 & 0 & 0 & 0 & 0 & 1 & 0 & 0 & 0 & 0 & 0 \\
0 & 0 & 0 & 0 & 0 & 0 & 0 & 1 & 0 & 0 & 0 & 0 \\
0 & 0 & 0 & 0 & 0 & 0 & 0 & 0 & 1 & 0 & 0 & 0 \\
0 & 0 & 0 & 0 & 0 & 0 & 0 & 0 & 0 & 1 & 0 & 0 \\
0 & 0 & 0 & 0 & 0 & 0 & 0 & 0 & 0 & 0 & 1 & 0 \\
0 & 0 & 0 & 0 & 0 & 0 & 0 & 0 & 0 & 0 & 0 & 1
\end{array}\right]-\left[\begin{array}{llllllllllll}
0 & 0 & 0 & 0 & 0 & 0 & 0 & 0 & 0 & 0 & 0 & 1 \\
0 & 0 & 0 & 0 & 0 & 0 & 0 & 0 & 0 & 0 & 0 & 1 \\
0 & 0 & 0 & 0 & 0 & 0 & 0 & 0 & 0 & 0 & 0 & 1 \\
0 & 0 & 0 & 0 & 0 & 0 & 0 & 0 & 0 & 0 & 0 & 1 \\
0 & 0 & 0 & 0 & 0 & 0 & 0 & 0 & 0 & 0 & 0 & 1 \\
0 & 0 & 0 & 0 & 0 & 0 & 0 & 0 & 0 & 0 & 0 & 1 \\
0 & 0 & 0 & 0 & 0 & 0 & 0 & 0 & 0 & 0 & 0 & 1 \\
0 & 0 & 0 & 0 & 0 & 0 & 0 & 0 & 0 & 0 & 0 & 1 \\
0 & 0 & 0 & 0 & 0 & 0 & 0 & 0 & 0 & 0 & 0 & 1 \\
0 & 0 & 0 & 0 & 0 & 0 & 0 & 0 & 0 & 0 & 0 & 1 \\
0 & 0 & 0 & 0 & 0 & 0 & 0 & 0 & 0 & 0 & 0 & 1 \\
0 & 0 & 0 & 0 & 0 & 0 & 0 & 0 & 0 & 0 & 0 & 1
\end{array}\right]\right]=0
$$

Since $\rho(z)=z^{12}(z-1)$ gives roots that lie within 0 and 1 , hence our method is zero stable. 


\section{NUMERICAL EXAMPLES}

Notation used in the table

ERA $\rightarrow$ Error in Areo et al. (2012)

ERB $\rightarrow$ Error in Badmus and Mishelia (2012)

\section{Problem 1}

We consider a linear first order ordinary differential equation

$y^{\prime}=x-y, y(0)=0,0 \leq x \leq 1, h=0.1$

Exact solution : $y(x)=x+e^{-x}-1$

This problem was solved by Areo et al. (2011) using block method of order seven. They adopted classical

RungeKutta method to provide the starting values. The result is shown in table 1

Table 1 showing results for problem 1

\begin{tabular}{|l|l|l|l|l|}
\hline $\mathrm{X}$ & Exact Result & Computed Result & $\begin{array}{l}\text { Error in our } \\
\text { method }\end{array}$ & ERA \\
\hline 0.1 & 0.004837418035959 & 0.00483741805555 & $1.9595(-11)$ & 0.000 \\
\hline 02 & 0.01873075307798 & 0.01873075311344 & $3.54623(-11)$ & 0.000 \\
\hline 0.3 & 0.04081822068171 & 0.04081822072989 & $4.81315(-11)$ & $6.0(-10)$ \\
\hline 0.4 & 0.07032004603563 & 0.07032004609377 & $5.80680(-11)$ & $2.0(-10)$ \\
\hline 0.5 & 0.10653065971263 & 0.10653065977831 & $6.56779(-11)$ & $7.0(-10)$ \\
\hline 0.6 & 0.14881163609402 & 0.14881163616533 & $7.13132(-11)$ & $1.0(-10)$ \\
\hline 0.7 & 0.19658530379140 & 0.19658530386669 & $7.52814(-11)$ & $8.0(-10)$ \\
\hline 0.8 & 0.24932896411722 & 0.24932896419507 & $7.78485(-11)$ & $2.0(-10)$ \\
\hline 0.9 & 0.30656965974059 & 0.30656965981984 & $7.92403(-11)$ & $9.0(-10)$ \\
\hline 1.0 & 0.36787944117144 & 0.36787941251113 & $7.96712(-11)$ & $4.0(-10)$ \\
\hline
\end{tabular}

Problem 2y' $x y, y(0)=1, h=0.1$

Exact solution: $y(x)=e^{\frac{1}{2} x^{2}}$

This problem was solved by Badmus and Mishelia (2011) using self-starting block method of order six, the result is shown in Table 2

Table 2 showing results for Problem 2

\begin{tabular}{|l|l|l|l|l|}
\hline $\mathrm{X}$ & Exact Result & Computed Result & $\begin{array}{l}\text { Error in our } \\
\text { method }\end{array}$ & ERB \\
\hline 0.1 & 1.00501252085940 & 1.0001252083353 & $2.6067(-11)$ & $5.29(-07)$ \\
\hline 02 & 1.02020134002675 & 1.0202013399419 & $8.4790(-11)$ & $1.77(-07)$ \\
\hline 0.3 & 1.04602785990871 & 1.0460278597221 & $1.8684(-10)$ & $8.99(-07)$ \\
\hline 0.4 & 1.08327067674958 & 1.0832870673239 & $3.5701(-10)$ & $3.09(-06)$ \\
\hline 0.5 & 1.13314845306682 & 1.1331485245627 & $6.1054(-09)$ & $1.91(-06)$ \\
\hline 0.6 & 1.19721736312118 & 1.1972173621060 & $1.0157(-09)$ & $4.48(-06)$ \\
\hline 0.7 & 1.27762131320488 & 1.2776213115603 & $1.6445(-09)$ & $1.02(-05)$ \\
\hline 0.8 & 1.37712776433595 & 1.3771277617200 & $2.6158(-09)$ & $7.74(-06)$ \\
\hline 0.9 & 1.49930250005676 & 1.4993024959457 & $4.1110(-09)$ & $1.44(-05)$ \\
\hline 1.0 & 1.64872127070012 & 1.6487212642939 & $6.4070(-09)$ & $2.93(-05)$ \\
\hline
\end{tabular}

\section{DISCUSSION OF THE RESULT}

We have considered two numerical examples to test the efficiency of our method. Problem 1 was solved by Areoet al. (2012). They proposed a hybrid method of order seven and adopted classical RungeKutta method to provide the starting values. The new method gave better approximation because the proposed method is self-starting and does not require starting values. Problem 2 was solved by Badmus and Mishelia (2012). They adopted self-starting block methods of order six. Our method gave better approximation because the iteration per step in the new method was lower than the method proposed by Badmus and Mishelia (2012)

\section{CONCLUSION}

We have proposed an order seven continuous hybrid method for the solution of first order ordinary differential equations. Our method was found to be zero stable, consistent and converges. The numerical examples show that our method gave better accuracy than the existing methods. 


\section{Reference}

[1] Areo, E.A, Ademiluyi, R.A and Babatola, P.O. (2011). "Three-step hybrid linear multistep method for the solution of first order initial value problems in ordinary differential equations", J.N.A.M.P,19,261-266

[2] Awoyemi, D.O, Ademiluyi, R.A and Amusegham,(2007). "Off-grid points exploitation in the development of more accurate collocation method for solution of ODEs", J.N.A.M.P. 12, 379-386

[3] Badmus, A.M and Mishehia, D.W (2011), "Some uniform order block methods for the solution of first ordinary differential equation", J. N.A.M. P, 19, 149-154

[4] Fatokun, J, Onumanyi, P and Serisena, U.V (2005), "Solution of first order system of ordering differential equation by finite difference methods with arbitrary". J.N.A.M.P, 30-40.

[5] Ibijola, E.A, Skwame, Y anKumleng G. (2011). "Formation of hybrid method of higher step-size, through the continuous multistep collation, American J. of Scientific and Industrial Research, 2(2), 161-1732)

[6] Salmon H. Abbas (2006). Derivation of a new block method similar to the block trapezoidal rule for the numerical solution of first order IVPs.Science Echoes, 2 10-24

[7] Salmon H. Abbas (2006). Derivation of a new block method similar to the block trapezoidal rule for the numerical solution of first order IVPs.Science Echoes, 2 10-24

[8] Yahaya, Y.A and Kimleng, G.M. (2007). "Continuous of two-step type method with large region of absolute stability", J.N.A.M.P, $11,261-268$

[9] Zarina B.I., Mohamed, S., Kharil, I and Zanariah, M (2005). "Block method for generalized multistep method Adams and backward differential formulae in solving first order ODEs, MATHEMATIKA, 25-33

[10] Zarina B.I., Mohamed, S., Kharil, I and Zanariah, M (2005)."Block method for generalized multistep method Adams and backward differential formulae in solving first order ODEs, MATHEMATIKA, 25-33 